\title{
IMPROVED INTERFEROMETRIC PROCEDURE WITH APPLICATION TO EXPANSION MEASUREMENTS
}

\author{
By James B. Saunders
}

\section{ABSTRACT}

The Fizeau interferometric method, as applied to thermal expansion measurements, has been studied in order to determine the source of some very serious errors and to find means of reducing such errors. Two types of errors were found to predominate. These may be termed (1) error caused by tilting of the spacers under mechanical forces, and (2) error caused by variations in air-film thickness at the contact points. Such errors often amount to 10 percent and may, under certain conditions, exceed in magnitude 50 percent of the value sought.

In this paper the two types of errors are considered in detail, and a modified procedure is given whereby the errors are reduced to a magnitude comparable to other unavoidable errors, such as those in reading the fringes and in determining the actual temperature of the specimens.

\section{CONTENTS}

I. Introduction

II. Indications of errors

III. Demonstration of tilting as a source of error

IV. Modified procedures

1. Correction of data

2. Elimination of tilting and surface-film effects

3. Weighting of spacer

V. Results showing the value of the weighted spacer procedure......... 188

VI. Necessary precautions

\section{INTRODUCTION}

A study of the variations in expansivity caused by differences in heat treatments or by minor differences in composition requires the measurement of small differences in the expansion of materials. Various differential methods are in use, but most of these require larger samples than are suitable for many types of investigations. When it is necessary to use very small samples, the Fizeau interferometric method ${ }^{1}$ is the only one available. The accuracy usually claimed for results ${ }^{2}$ obtained by this method is more than adequate; however, when the method is applied in the usual manner, ${ }^{3}$ the required accuracy cannot always be assured. A modification of the Fizeau method used by Peters ${ }^{4}$ would seem ideal, but it too has proved unsatisfactory for the purpose now in view.

When the interferometer is applied in the form mentioned above, a progressive change is usually observed in the width and orientation

\footnotetext{
1 L. Fizeau, Ann. phys. 128, 564 (1866).

H. G. Dorsey, Phys. Rev. 25, 88 (1907); H. M. Randall, Phys. Rev. 20, 10 (1905); H. D. Ayers, Phys. Rev. 20, 38 (1905); J. B. Austin, Physics 3, 250 (1932).

${ }^{3}$ C. G. Peters and C. S. Cragoe,BS Sci. Pap. 16, 449 (1920) S393.

1 C. G. Peters, J. Wash. Acad. Sci. 9, 281 (1919).
} 
of the fringes. This change can only be caused by a tilting of one interferometer plate with respect to the other. Such a tilting normally occurs if three spacers of different thermal expansivities are used to separate the plates, but it also often occurs in the absence of differential expansions and indicates, therefore, the presence of spurious displacements.

The present study was undertaken in order to determine the source of these spurious displacements and also to find means of eliminating them. The causes of these displacements were found to be twofold: (1) Changes in surface-film thicknesses at the points of contact between the plates and spacers, and (2) tilting of the individual spacers when not rigidly attached to each other. In some cases the magnitude of the resulting errors was found to exceed 50 percent of the true value of the expansion; but it was also found that either they can be eliminated from the initial observations by certain precautions, or their magnitude can be determined for the purpose of applying corrections to the observations. By following either procedure, the precision claimed for the interferometric method of expansion measurements can be fully attained.

\section{INDICATIONS OF ERRORS}

Before it was realized that the errors could be effectively eliminated from results furnished by the interferometric method, it was always found necessary to take many sets of data on each sample in order to make sure that the results were fairly representative of the values sought. Although changes in the fringe pattern were quite obvious, it has not been generally realized, heretofore, that these erratic fringe shifts were indicative of spurious displacements which resulted in large errors in the observed data. In fact, errors of this type are prevalent in many of the published data where the interferometric method has been used.

In the course of a test, the number of fringes between 2 supporting spacers of the same material is frequently observed to change by as much as 10. In previous procedures this has been assumed to be unimportant. This change indicates, however, that for the temperature interval used, 1 spacer increases the separation of the interferometer plates by an amount equivalent to 10 fringes more than the increase caused by the other spacer. If the spacers expand equally, some spurious change must have taken place which produces a difference in plate separation equivalent to 10 fringes. Usually, on repeating the test without disturbing the specimens (spacers), this observed difference is closely duplicated. However, when the setup is removed from the furnace and reassembled, this apparent differential expansion of the spacers is usually appreciably different.

In many cases a fringe configuration that has been changing more or less slowly with the temperature will suddenly take on a new form which is usually more like the initial configuration. When this occurs, the shift of the fringes past any point represents a corresponding jump in the average separation of the interferometer plates. Other observers ${ }^{5}$ have noted such changes, but apparently they have failed to realize the magnitude of the associated errors.

' See, for example, H. G. Dorsey, Phys. Rev. 25, 88 (1907). 


\section{DEMONSTRATION OF TILTING AS A SOURCE OF ERROR}

A warping of the spacers, caused possibly by their failure to slide on the lower plate, which usually expands differently, was first believed to be the cause of the errors mentioned above. The action of the fringes agrees very well with such an assumption. They change slowly in width and orientation as the spacers expand; then, as if some constraining force suddenly disappeared, they return approximately to their original configuration.

To test this possibility of warping, three specially shaped spacers were made from a specimen of optically homogeneous glass (dense barium crown, NBS melt $480 ; n_{D}=1.6225, \nu=56.7$ ). The expansivity of each spacer was then determined separately (by a procedure described in section IV-3 of this paper) and the results showed the thermal expansions of the spacers to be equal within the required limits of accuracy. Each of these spacers (fig. 1, A) was made from two parts (fig. 1, B) by cementing the base of a small conical part on top of a relatively broad, low, stool-like tripod part. The three feet of each tripod were equally spaced, the top was polished flat, and the legs were made almost equal in length so that when the tripod was standing on the flat surface of an interferometer plate the top was almost parallel to it. The height of each spacer was then adjusted almost to equality with that of the others by grinding off the conical tips. The tip of each spacer was vertically above the center (median point) of the equilateral triangle formed by the three feet of the tripod. The four supporting areas (three feet and conical tip) of each spacer were made small by pointing.

On mounting the three spacers between two fused-quartz interferometer plates, easily readable fringes were obtained between the quartz plates and also between the top of each stool and the upper quartz plate. The primary fringe system is represented as set $D$ in figure 2 , and the three secondary sets, formed by the stool tops and the upper plate, by $A, B$, and $C$. On heating this assembly from $25^{\circ}$ to $250^{\circ} \mathrm{C}$, the fringes changed gradually from the numbered configuration represented by figure $2(A)$ to that by $2(B)$, and all fringes remained straight regardless of changes in width and orientation. If the changes in the fringe system had been caused by a warping of the tripod part of any spacer, the fringes over this spacer would have become curved. Consequently, it is concluded that no warping occurred.

Since the construction of these particular spacers is such that the air-film errors cannot be large, the only other cause to which one can attribute the gross errors under discussion is a tilting of one or more of the spacers, where the tilting is the result of one or two feet of a spacer being lifted from the base plate. As will be seen, this action was definitely established as causing most of the errors.

When viewing an interferometer fringe system with collimated light, the number of fringes between any two points is a direct indication of the difference in the distance between the interferometer plates at those points. If the separation is known at one point, it may easily be determined at any other by adding or subtracting the difference in separation of the plates at the two points. The magnitude of this difference is known from the number of fringes observed between 
the points, and its sign is determined by noting the direction of fringe motion when the plate separation is increased or decreased.

Since it has now been shown that the spacers do not warp or expand differentially, any relative rotation of the interferometer plates must result from a changed separation between the spacers and plates at some of their contact points. Such changes in the separation between the spacers and the top plate will always be comparatively small, since there is only one contact point for each of the three spacers; nevertheless, this change at any spacer will produce an equal change in the separation of the plates at that point. Since there are three contacts between a spacer and the lower plate, any change in separation at one of these contact points produces (for the type of separators used) only one-third as much change in the total separation of the interferometer plates at the fiducial point over the center of the spacer. The changes at some of the lower points are, however, much larger than at the upper points. This is because the spacer can tilt up on one or two of its feet, causing at least one foot to leave the base plate entirely. The cause of this tilting is assumed to be as follows: In general, one spacer is more stable than either of the others, due to either a greater load, a greater spread of its feet, or both. Also, one foot of the most stable spacer will usually bear a greater load than either of its other two feet. However, a differential expansion between this spacer and the lower plate will force two of its feet to slip. This results in a sidewise motion of the spacer which, in turn, causes a horizontal motion of the top plate. This action on the top plate generally causes the other two spacers to tilt and the motion is usually in such a direction as to force two feet of each of these spacers to be lifted from the base plate. As soon as this condition develops, the top plate usually begins to rotate horizontally about the contact point with the stable spacer, while the unstable spacers rotate similarly about their own contacts with the base plate. This combination of motions continues until a third foot of the unstable spacers comes into contact with the base plate. The motions are now arrested until differential expansion causes further tilting.

To study the action and magnitude of the tilting, reference marks (fig. 2) were placed on the top surface of each stool vertically above the contacts of its three feet with the lower plate. These marks, which correspond to the points of contact between spacers and lower plate, are designated by the trios: 11,12 , and 13 for spacer $A ; 21$, 22 , and 23 for spacer $B$; and 31,32 , and 33 for spacer $C$. The three supporting points of the top plate, indicated by 10,20, and 30 for spacers $A, B$, and $C$, respectively, were marked on the upper surface of the top interferometer plate and are at the median points of the triangles formed by the above three sets of points. Thus, there are 4 reference points in each of the fields $A, B$, and $C$; and all 12 points may be considered as being within field $D$. The $D$-fringes are obscured at the reference marks by the spacers, as are also the $A, B$, and $C$-fringes at the points 10,20 , and 30 , respectively. The fringe positions with respect to these points, however, can be read very accurately with the aid of a straight filament which lies in the focal plane of the observing instrument and is rotatable about the optic axis. The instrument employed could be moved horizontally as a whole so as to bring such a filament over the image of any point in the field of view. 


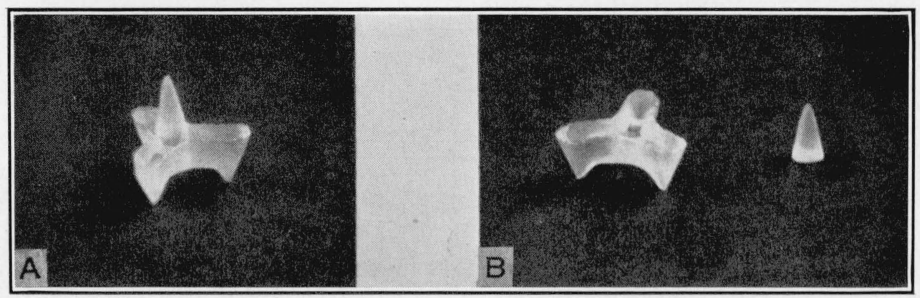

FIGURE 1.-A form of specimen used for the demonstration of tilting.

$A$, complete specimen after assembly, and $B$, tripod (or stool) with flat and polished top, and cone-shaped piece, the base of which was sealed to top of tripod. 
The interferometer system shown in figure 2 was properly adjusted in an electric furnace, ${ }^{6}$ and data were taken at the points 10,20 , and 30 as the furnace was heated from room temperature $\left(25^{\circ} \mathrm{C}\right)$ to about $250^{\circ} \mathrm{C}$. These data were used to compute the apparent expansion of the three spacers for this interval, and the results shown in table 1 , column 2, indicate that the three spacers have different apparent expansivities. The test was repeated a second, third, and fourth time without disturbing the interferometer, and columns 3, 4, and 5 of table 1 show that the results obtained by these three tests for the expansion of spacer $A$ were practically identical. However, the results for the same spacer on the first or preliminary test (column 2) differ somewhat from them.

TABLE 1.-Expansivity of a glass at $100^{\circ} \mathrm{C}^{1}$

\begin{tabular}{|c|c|c|c|c|}
\hline \multirow{2}{*}{ Spacer } & \multicolumn{4}{|c|}{ Observed expansivity $\times 10^{4}\left(\right.$ at $100^{\circ} \mathrm{C}$ ) } \\
\hline & 1st run & $2 \mathrm{~d}$ run & $3 \mathrm{~d}$ run & 4 th run \\
\hline $\begin{array}{l}A \\
B \\
C\end{array}$ & $\begin{array}{l}7.37 \\
7.87 \\
7.50\end{array}$ & $\begin{array}{l}7.34 \\
8.00 \\
7.68\end{array}$ & $\begin{array}{l}7.34 \\
7.97 \\
7.72\end{array}$ & $\begin{array}{l}\text { 7. } 33 \\
\text { 7. } 97 \\
\text { 7. } 70\end{array}$ \\
\hline
\end{tabular}

1 This table illustrates the necessity of making a preliminary run (1st run, column 2) before consistent data can be obtained. The results for $B$ and $C$ after the first run are fairly consistent but not so consistent or accurate as those of $A$, for reasons described later.

While the results for spacers $B$ and $C$ are fairly consistent for the four runs, yet they are much less so than for spacer $A$. Furthermore, the results for the three spacers differ greatly among themselves. These data indicate that spacer $A$ was the most stable and that its greater stability caused it to remain fixed in position, whereas the others were forced to tilt by differential expansion. Any tilt of the spacers will necessarily cause their apparent expansivities to be too great. Since the tilt would, in general, be different for different spacers, the computed expansions could not be expected to agree. The slight difference between the results of the first test and those of the later tests suggests also that there was an initial surface film trapped between the contact points, and that this film disappeared during the first heating, or at least was reduced to a minimum and remained constant throughout the remaining tests. ${ }^{7}$ The differences between the second, third, and fourth runs then appear to be caused almost entirely by tilting.

After the tests described above, a fifth heating was given the furnace. The initial fringe configuration (at room temperature) was observed to be that shown in figure $2(A)$. After completing the observations at room temperature, the interferometer assembly was heated and the changing order of the fringes at each of the spacers was recorded at frequent intervals. When the temperature reached $250^{\circ} \mathrm{C}$, another set of readings was taken, and the observed fringe configuration is indicated in figure $2(B)$. The temperature was held constant while the data (table 2 ) for each of these two drawings were taken. The differences in the various fringe systems allow a computation of the change in separation of the interferometer surfaces at each of the several reference points. 


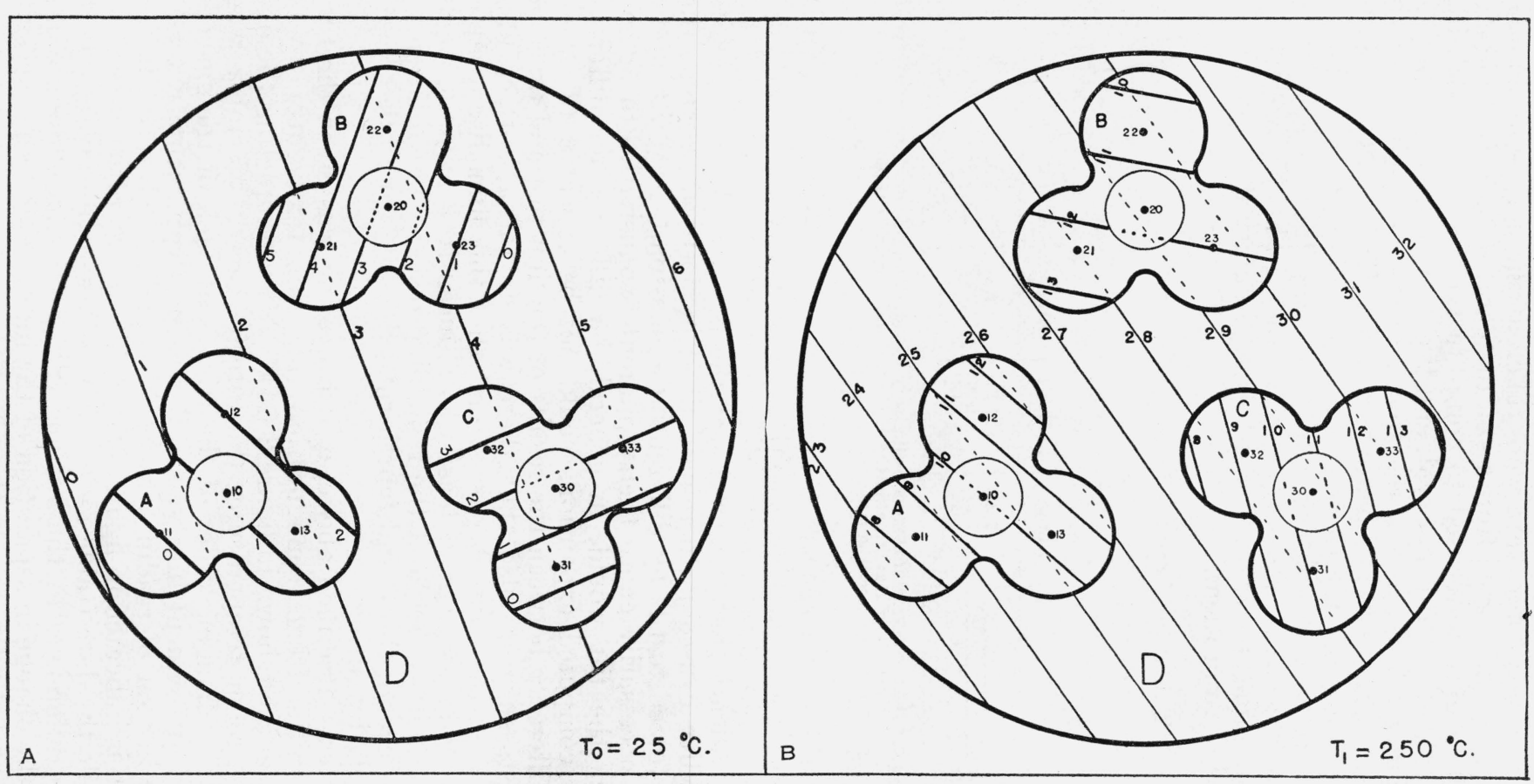

FiguRe 2.-Diagrammatic representation of the fringes observed in the multiple interferometer field before and after a change in temperature.

The straight lines represent the observed fringes and are identified by the associated numbers. These numbers indicate the fringe count in relation to the fringes, which The small dots represent the reference points over the various contact points. These points are designated by the associated numbers. The curved lines represent the outlines of the various fields. 
TABLE 2.-Fringe readings

[Data taken from figure 2]

\begin{tabular}{|c|c|c|c|c|c|}
\hline \multicolumn{2}{|c|}{ Temperature } & \multicolumn{2}{|c|}{$25^{\circ} \mathrm{C}$} & \multicolumn{2}{|c|}{$250^{\circ} \mathrm{C}$} \\
\hline Spacer & $\begin{array}{l}\text { Reference } \\
\text { point }\end{array}$ & $\begin{array}{l}\text { Primary } \\
\text { field }\end{array}$ & $\begin{array}{l}\text { Secondary } \\
\text { field }\end{array}$ & $\begin{array}{l}\text { Primary } \\
\text { field }\end{array}$ & $\begin{array}{l}\text { Secondary } \\
\text { field }\end{array}$ \\
\hline$A_{-}$ & $\begin{array}{l}10 \\
11 \\
12 \\
13\end{array}$ & $\begin{array}{l}1.35 \\
0.65 \\
1.65 \\
1.80\end{array}$ & $\begin{array}{l}1.10 \\
0 . \\
2.00 \\
1.40\end{array}$ & $\begin{array}{l}24.70 \\
23.50 \\
25.35 \\
25.20\end{array}$ & $\begin{array}{r}10.00 \\
8.40 \\
11.25 \\
10.35\end{array}$ \\
\hline$B_{--}$ & $\begin{array}{l}20 \\
21 \\
22 \\
23\end{array}$ & $\begin{array}{l}3.75 \\
3.05 \\
4.00 \\
4.20\end{array}$ & $\begin{array}{l}2.75 \\
3.90 \\
3.35 \\
1.00\end{array}$ & $\begin{array}{l}29.15 \\
28.00 \\
29.80 \\
29.65\end{array}$ & $\begin{array}{l}11.65 \\
12.40 \\
10.55 \\
12.00\end{array}$ \\
\hline$C_{\ldots} \ldots$ & $\begin{array}{l}30 \\
31 \\
32 \\
33\end{array}$ & $\begin{array}{l}\text { 4. } 25 \\
\text { 4. } 00 \\
3.80 \\
4.95\end{array}$ & $\begin{array}{l}1.75 \\
0.45 \\
2.85 \\
1.90\end{array}$ & $\begin{array}{l}28.80 \\
28.15 \\
28.25 \\
30.00\end{array}$ & $\begin{array}{r}10.65 \\
10.15 \\
9.25 \\
12.50\end{array}$ \\
\hline
\end{tabular}

If it is assumed that all spacers expand equally, that all feet contact the base plate at the initial reading, and that each spacer contacts each plate at one or more points at all times during a test, the magnitude of all tilts may be computed from the data of table 2. The change in the number of fringes between any two points in a secondary fringe field over any stool gives the rotation of the top plate with respect to that stool top about a horizontal axis perpendicular to a line joining the two points; similarly, the corresponding change in field $D$, between the same two points, gives the rotation of the top plate with respect to the lower interferometer plate. The difference between these two rotations is the rotation of the stool with respect to the bottom plate; and this rotation is, in turn, proportional to the difference in the separation between the feet of the spacer (stool) and the lower plate at these points. If we consider the set of fringes marked $A$ in figure 2, and let $T_{11}, T_{12}$, and $T_{13}$ represent the changes in separation between this spacer and the lower plate at the points 11 , 12, and 13, respectively, we have the following relationships:

$$
\left.\begin{array}{l}
T_{12}-T_{11}=\Delta_{12},{ }_{11}, \\
T_{13}-T_{11}=\Delta_{13},{ }_{11}
\end{array}\right\}
$$

where the $\Delta$ 's represent the difference between the change in number of fringes between the points, indicated by the subscripts, in field $A$ and the corresponding fringe change in field $D$. Since one of the $T$ 's in eq 1 must be zero, according to the above assumptions, these equations are easily solved by placing the smallest $T$ equal to zero. The magnitude of the two $\Delta$ 's gives the order in magnitude of tilts $T_{12}$ and $T_{13}$, and the magnitude of either $\Delta$ gives the order in magnitude of the two T's appearing in the same equation as the $\Delta$ considered.

In this manner all tilts were determined (see table 3 ), and the results of several such sets of data indicated that at least one spacer does not tilt and that the others usually do. All results agreed perfectly with the assumption as to the nature of the tilting. 
TABLE 3.-Computed tilts (in fringe equivalents)

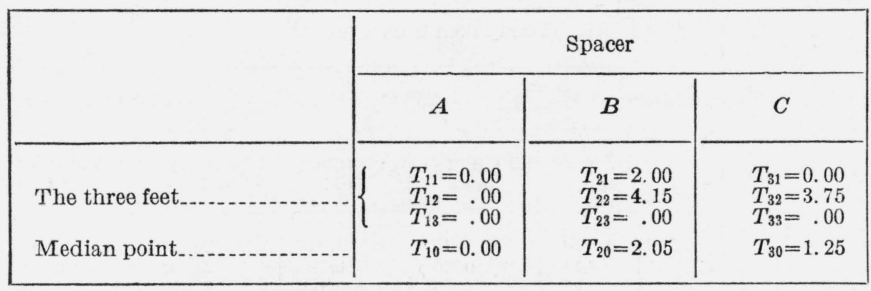

\section{MODIFIED PROCEDURES}

\section{CORRECTION OF DATA}

It has been found that when an interferometer (of the type mentioned above) is heated, at least one of the three spacers does not tilt, and that a preliminary heating practically frees this spacer from the effects of surface film changes. Under these conditions the error caused by tilting and by changing surface films at the contacts of the other spacers may be evaluated and used as a correction to data taken at any chosen reference point.

In presenting this method of corrections, which has been used successfully, it will be assumed that the observations requiring the corrections are taken at the median point of an equilateral triangle formed by the contact points on which the top interferometer plate rests. The method is not restricted to cases meeting this condition. It has been introduced merely to simplify the discussion and to facilitate the computations of the corrections. If all spacers properly contact the interferometer plates in the beginning, and if at least one spacer does so at each later observation, the corrections for the observations at the median point may be obtained from the change in the number of fringes between the contact point of the most stable spacer with the top plate and the corresponding points of the other two spacers. If we represent these points by 10,20 , and 30 (see fig. 2) and assume point 10 to be over the most stable spacer, then the correction to be supplied is $1 / 3\left(\delta_{10,20}+\delta_{10,30}\right)$ where $\left(\delta_{10,20}\right)$ represents the change in the number of $D$-fringes between points 10 and 20, and where $\left(\delta_{10,30}\right)$ represents the corresponding change between points 10 and 30. This correction is to be subtracted from the observed order of the fringes read at the median reference point, and the result is what would have been observed at this point had there been no tilting or changes in the films between the spacers and the plates. The most stable spacer is easily identified as the one giving the least apparent expansion. If there are two such spacers, either one may be chosen with no difference in the results.

\section{ELIMINATION OF TILTING AND SURFACE-FILM EFFECTS}

In order to eliminate tilting, the spacers supporting the top plate must be bound together in one rigid unit. To produce this type of support, a spacer was made in the form of a ring, ${ }^{8}$ which was so cut that it rested with three feet on the lower plate and supported the top plate at three points, one over each of the feet. The use of this

\footnotetext{
- BS J. Research 11, 799 (1933) RP626.
} 
type of specimen greatly reduces the change in fringe configuration during a test, although small but troublesome changes still remained.

In adjusting these rings so that readable fringes were produced, the ring was finished on a flat stone, increased pressure being applied to the points where the height was too great. This treatment gave the spacers appreciable supporting areas which were relatively flat. To check the supposition that the small troublesome changes in fringe configuration were caused by variations in the thickness of the surface films in these areas between the spacers and plates, the three surfaces supporting the top plate (each having an area of approximately $1 \mathrm{~mm}^{2}$ ) were polished sufficiently flat to produce distinguishable fringes between them and the top plate. This added three small but sharp sets of fringes to the primary field between the plates, which could be easily observed although they were usually very far from being straight and uniform in width. On heating this interferometer system to $220^{\circ} \mathrm{C}$, the thickness of the films in the contact areas was observed to decrease by as much as 16 fringes (8 wave lengths of yellow helium light). Heating to higher temperatures, followed by cooling to room temperatures, always failed to produce further appreciable changes at one of the contact points. Even repeated heatings and coolings failed to produce further changes. At the other two points, however, fluctuations in the film thickness were always present after the first heating to $220^{\circ} \mathrm{C}$. The use of plates having the same expansivity as the specimens themselves greatly reduces this fluctuation but does not eliminate it. A further reduction is obtained by decreasing the temperature gradients between the plates and specimens and also by pointing the supports. This suggests that slippage at the contact points causes much of the change in surface film thickness after the first heating.

In accounting for these differences in stability, it was assumed that the first heating developed a condition in which the friction at one of the points was sufficient to force all of the slippage to take place at the other two points. No doubt similar variations occur in the film thicknesses at some of the contacts between the ring and the lower plate.

With the unit type of specimen (all three supporters of the top plate, bound rigidly together) none of the tilting mentioned in section III is observed, but the surface film effects are greater if the supporting areas of this spacer are relatively large. The correction method of the previous section is still applicable and in general the correction is smaller for this type of specimen.

If the reference point can be chosen over the contact point which is free from both tilting and surface film effects, then there is no such correction to be applied, and the resultant data are practically free from these errors.

\section{WEIGHTING OF SPACER}

Even though the errors mentioned above can be determined with fair accuracy, their direct elimination is always desirable. This elimination is accomplished by choosing the reference point at the proper place, which is easily located during the test as shown in the previous section. For practical application it becomes necessary to predetermine this point. This is done by increasing the stability of one spacer or support, in the case of a unit-type spacer. This favoring is accom- 
plished by shifting the center of the top interferometer plate towards the chosen spacer until it bears most of the weight of the top plate, or an auxiliary weight may be placed on the top plate near this spacer. A U-shaped weight is convenient since it may be so placed as to add load to the chosen spacer only, without obscuring the fringes there.

In using this procedure, it was found that the chosen spacer should carry several times the load of either of the other two, and that under this condition the data obtained at an observation point over the weighted spacer appeared to be practically free from errors caused by tilting and by changing surface films. That is, after the preliminary heating of the specimens, the data seemed to be as reliable as an interferometric method is capable of yielding.

Obviously, the instability of the other spacers still produces changes in the fringe configuration, but as long as the fringes are broad enough for observation such changes do not affect the data obtained at the weighted spacer. To aid in maintaining the required fringe breadth, it is found advisable to weight the tallest spacer, since in that case any tilt usually leaves the fringes broader than they would have been with the same tilt if one of the shorter spacers had been weighted. Even then the fringes often get too narrow and the observer will have to rap gently on the furnace (a procedure which usually restores the fringes to their original width without introducing error in the data) in order to proceed with the observations. As stated before, the use of interferometer plates that have nearly the same expansion as that of the specimens greatly reduces such changes in the width of the fringes.

If, when three individual spacers are used, the triangular base of the weighted spacer is small compared to those of the other two, or if the contact point of this spacer with the top plate is not sufficiently near the center of the base triangle, the stability of the spacer is then relatively low, and the pull of the other two spacers may ultimately overcome it. To insure stability of the weighted spacer, its base should be at least as large as that of either of the other two. It should, however, not be too large, since increasing its size also increases the slippage of its feet produced by differential expansion and the tilt imposed on the other spacers.

\section{RESULTS SHOWING THE VALUE OF THE WEIGHTED SPACER PROCEDURE}

To illustrate the improvements obtained by using the weighted spacer procedure instead of the customary one, 12 samples were taken from a piece of optically homogeneous glass (medium flint, NBS melt $\left.457 ; n_{D}=1.6264 ; \nu=36.7\right)$ and tested as follows: From this set of samples three were selected as spacers and shaped so as to give good broad fringes when placed between two interferometer plates. Of these spacers the one designated by $n_{1}$ bore approximately threefourths of the total weight of the top plate. This interferometer assembly was placed in the furnace and expansion data were taken on three successive runs from room temperature to $250^{\circ} \mathrm{C}$. The three contact points of the spacers with the top plate were selected as reference points, at each of which expansion data were taken as described by Peters. ${ }^{9}$

○ J. Wash. Acad. Sci. 9 [10], 281 (1919). 
The interferometer was then removed from the furnace, and the load of the top plate shifted to another spacer, designated by $n_{2}$. Data for three runs were again taken at the three reference points. For a third set of similar data, spacer $n_{3}$ bore most of the weight of the top plate. From the data of these tests the expansivity at $100^{\circ} \mathrm{C}$ was computed for each run and for each spacer, as shown in table 4. In computing the accepted expansion, only the results obtained at a weighted reference point and after a preliminary heating were found to meet the desired precision. The accepted values are italicized and are averaged in the bottom row. The results show the expansion of each spacer to be very near $8.28 \times 10^{-6}$, and that the expansions of the three spacers differ very little, if any, from each other. The various values obtained by the different tests on the unweighted specimens are seen to differ broadly among themselves, and the same is also true for the results obtained by the first or preliminary tests on all specimens.

TABLE 4.-Expansivity of a glass (medium flint) at $100^{\circ} \mathrm{C}^{1}$

\begin{tabular}{|c|c|c|c|c|c|}
\hline \multirow[t]{2}{*}{ Spacer weighted } & \multirow{2}{*}{$\begin{array}{l}\text { Runs for } \\
\text { each ar- } \\
\text { rangement } \\
\text { of spacers }\end{array}$} & \multicolumn{4}{|c|}{$\begin{array}{l}\text { Expansivity } \times 10^{4} \text { of spacers }\left(n_{1}, n_{2} \text {, and } n_{3}\right) \text { as } \\
\text { determined from observations taken directly } \\
\text { over each of them }\end{array}$} \\
\hline & & $n_{1}$ & $n_{2}$ & $n_{3}$ & Average \\
\hline $\begin{array}{l}n_{1 \ldots-} \\
n_{2 \ldots-} \\
n_{3 \ldots-}\end{array}$ & 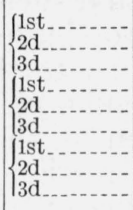 & $\begin{array}{l}\text { 8. } 26 \\
8.29 \\
8.27 \\
8.31 \\
8.25 \\
8.26 \\
7.95 \\
8.22 \\
8.26\end{array}$ & $\begin{array}{l}8.44 \\
8.54 \\
8.45 \\
8.29 \\
8.28 \\
8.27 \\
8.22 \\
8.26 \\
8.25\end{array}$ & $\begin{array}{l}8.25 \\
8.42 \\
8.46 \\
8.26 \\
8.66 \\
8.72 \\
8.30 \\
8.27 \\
8.29\end{array}$ & $\begin{array}{l}8.32 \\
8.42 \\
8.39 \\
8.29 \\
8.40 \\
8.42 \\
8.16 \\
8.25 \\
8.27\end{array}$ \\
\hline A verage of values in italic.... & - & 8. 280 & 8. 275 & 8. 280 & 8.278 \\
\hline
\end{tabular}

1 The figures in italic are those procured on weighted spacers after the preliminary (1st) runs.

After finding that the expansivities of the three spacers were so nearly identical that the changing fringe configurations, together with the resultant erratic apparent expansions of the unweighted spacers, could not be the result of actual differences in expansion, similar tests were then extended at the usual heating rate to the range of rapid expansion. However, instead of reading at three points, as above, the top point of the weighted spacer and the median point of the triangle formed by the three spacers were chosen as reference points. These points were selected for the purpose of comparing the results obtained by the weighted spacer procedure with those by the usual method, where the reference point is generally chosen near the center of the triangle formed by the three supports of the top plate. The results are represented in figure 3 by solid dots. Curve $A$ was computed from data taken at the point over the weighted spacer. The other dotted curves $\left(A^{\prime}, A^{\prime \prime}\right.$, and $\left.A^{\prime \prime \prime}\right)$ represent the data taken at the median point and show the effects of three possible interpretations of a sudden jump observed in the fringe configuration.

During this heating to the rapid expansion range, there were, at the beginning, 2.9 fringes crossing the triangle formed by the three spacers and 1.6 fringes between the two reference points. As the heating progressed, the fringes broadened until at $91^{\circ} \mathrm{C}$ there was only 0.2 
fringe between the reference points. Just before reaching this temperature, the fringes began to vibrate and change width as they rotated back and forth about the point where the top plate was supported by the weighted spacer. This signified that the rising of the top plate at this point was caused only by the uniform expansion of the weighted spacer, whereas it was bobbing erratically at other points. After a few moments the vibration stopped, leaving the fringe configuration as it was at the beginning of the heating; that is, with 2.9 fringes crossing the triangle and 1.6 fringes between the two reference points. Obviously, there had been an abrupt shift of 1.4 fringes at the median reference point as the result of one or both unweighted spacers having changed from a tilted to a relatively stable position.

The fringe reading at the median point was 9.3 just before the vibration started and $n+0.9$ ( $n$ being some integer) immediately after it stopped. If observations had been made only at the median point, the value for $n$ would not have been known, and the observer would probably have chosen either 8 or 9 as its value. If $n=9$, then the plate jumped up 0.3 of a wave length ( 0.6 fringe), and $A^{\prime \prime \prime}$ is the proper course of the curve. If $n=8$, the plate fell 0.2 of a wave length $(0.4$ fringe) and the proper curve follows $A^{\prime \prime}$. The slope of the curve before, and the position of the next point after the jump, would seem to favor curve $A^{\prime \prime \prime}$ as being the proper one to choose. On the other hand, the value $n=8$, and consequently curve $A^{\prime \prime}$, might seem the more probable because of a belief that such jumps are almostinvariably caused by a sudden sinking of the top plate. By having taken observations simultaneously at a point where the fringes did not jump, in addition to the readings at the median point, one can be certain of the corrections for the readings taken at the median point both before and after such a jump. These corrections are obtained from a consideration of the change in the number of fringes between the two reference points. Thus the correct value to be assigned to $n$ was found to be neither 9 nor 8 , but 7 , giving curve $A^{\prime}$ as the real curve for the change in distance between the interferometer plates at the median reference point. That is, the jump at this point"was from 9.3 down to 7.9 , a jump of 1.4 fringes, which was the sudden change"observed in the number of fringes between the two reference points.

After these tests the remaining nine samples were similarly tested, three at a time, to the rapid expansion range of this glass. In each case the samples were given a preheating to $250^{\circ} \mathrm{C}$ and allowed to cool back to room temperature. The furnace was then given a slight shock to rectify any remaining tilt that might have developed during the preliminary treatment. As before, two curves were obtained from each test, one giving the real expansion of the weighted spacer, and the other giving the average apparent expansion of all three spacers. The real expansion values ${ }^{10}$ for these three sets of spacers are plotted in figure 3 as curves B, C, and D. Obviously, these curves practically coincide with curve $A$.

The curves for the apparent expansions, as obtained from the data taken at the median points, showed certain peculiarities which are similar to those often appearing in curves procured by other investigators ${ }^{11}$ who have used the median point between three individual

\footnotetext{
${ }_{10}$ Because of crowding, only every fourth point is plotted in curves $A, B, C$, and $D$ of figure 3; all points are, however, indicated in figure 4.

11 This knowledge was gained through private communications.
} 
spacers in procuring expansion data. That is, one or more rather definite changes in slope ${ }^{12}$ have often appeared at certain points in the range between $100^{\circ}$ and $400^{\circ} \mathrm{C}$, and abrupt irregularities or slight jumps in the trend of the observations have also been noted occasionally. These changes in slope probably develop from a progressive tilting of one or two of the spacers followed by a slow readjustment. The readjustment may be caused either by a gradual slippage at one or more of the contact points or by a slow rotation of the tilted spacers

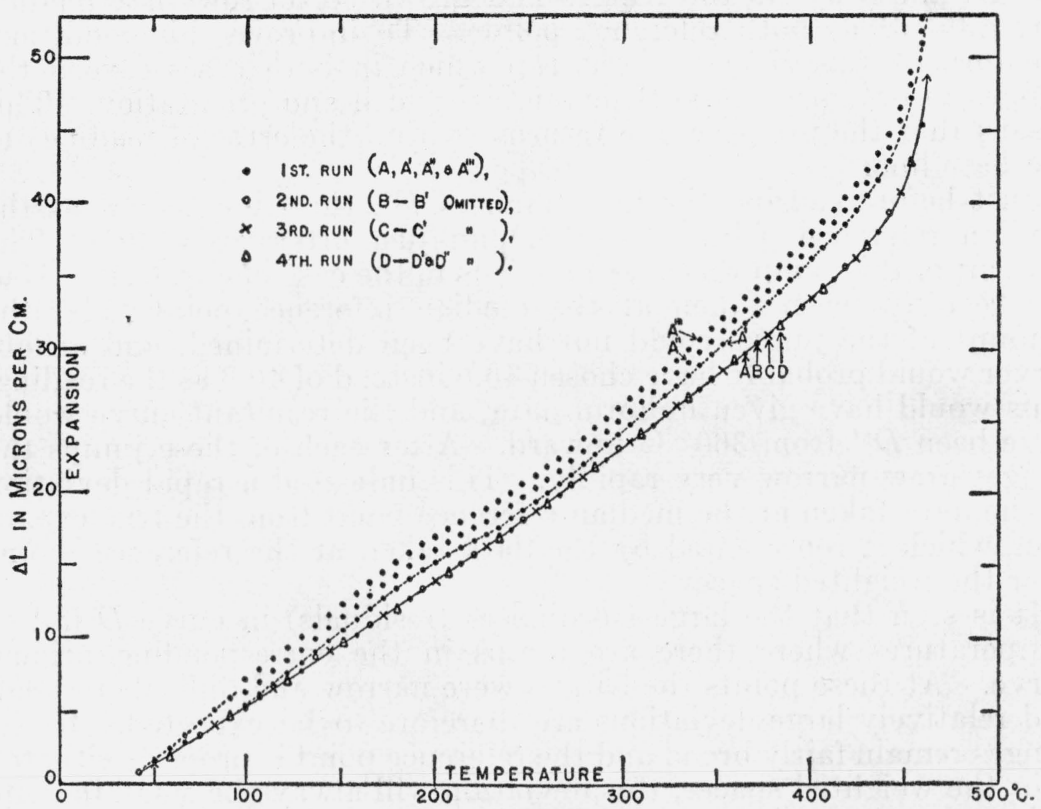

FIGURE 3.-Expansion curves, showing uncertainty in data taken at median reference point.

The curves $A^{\prime \prime}$ and $A^{\prime \prime \prime \prime}$, represented throughout by solid dots, indicate the different results obtained by a failure to apply proper corrections for tilting. The curve represented by solid dots and dashes indicates the result of applying the proper correction at one temperature (near $90^{\circ} \mathrm{C}$ ) and not elsewhere. The composite curve is based on every fourth observation of four tests, each on different sets $(A, B, C$, and $D)$ of separators. These observations were all so obtained that corrections for tilting were unnecessary, as shown in the text.

about these points. Such changes in slope are particularly evident in curve $A^{\prime}$, figure 3 , between $200^{\circ}$ and $300^{\circ} \mathrm{C}$. The more abrupt irregularities noted (indicated by discontinuities at vertical arrows in the curves of fig. 4) are caused by sudden readjustments after a progressive tilting.

In figure 4 the residuals are plotted for all curves obtained from the four sets of spacers. In obtaining these residuals, the proper ordinate of the best curve obtainable for fitting the preferred data (that taken at the points over the weighted spacers) was subtracted from the data observed at all reference points and the difference plotted on an enlarged scale. In this plot the ordinates of the various curves correspond very closely to the errors in the data. Curves $A, B, C$,

12 The same type of discontinuities were also obtained by Klemm and Berger, Glastech. Ber. 5, 405 (927), and by Turner and Winks, J. Soc. Glass Tech. 14, 84 (1930), using entirely different methods of measurements.

$152687-39-13$ 
and $D$ are the residuals for the data taken at the reference point over the weighted spacers. As seen, the deviations in these curves are small compared to those in $A^{\prime}, B^{\prime}, C^{\prime}$, and $D^{\prime}$, which are the residual curves for the data taken at the median points. There are no sudden breaks in curves $B^{\prime}$ and $C^{\prime}$ such as that in $A^{\prime}$ at $91^{\circ} \mathrm{C}$. These curves were smooth and the data appeared good, but when compared to the companion curves $B$ and $C$, they are obviously quite erroneous. Curve $D^{\prime}$ has an abrupt break at or near $360^{\circ} \mathrm{C}$ and another at $440^{\circ}$ C. At these points the fringes had grown so narrow that reading was difficult at both reference points. To improve this condition, the furnace was given a slight rap which in both cases caused the fringes to broaden out to their original width and orientation. This means that the proper curve returns (within the error of reading) to the base line.

Just before causing the first jump in $D^{\prime}$, the fringe count at the median reference point was 46.5 , and just afterwards, 40.6. The amount of this jump was determined as in the case of curve $A^{\prime}$. Had the readings been taken at the median reference point only, the amount of the jumps could not have been determined, and an observer would probably have chosen 46.6 instead of 40.6 as the reading. This would have given uniform data, and the resultant curve would have been $D^{\prime \prime}$ from $360^{\circ} \mathrm{C}$ upward. After each of these jumps the fringes grew narrow very rapidly. This indicated a rapid deviation of the data taken at the median reference point from the true expansion, which is represented by the data taken at the reference point over the weighted spacer.

It is seen that the largest ordinates (residuals) in curve $D$ fall at temperatures where there are jumps in the corresponding primed curve. At these points the fringes were narrow and difficult to read, and relatively large deviations are therefore to be expected. If the fringes remain fairly broad and the reference point is properly situated over the weighted spacer, this deviation will always be small in spite of jumps and rotations.

\section{NECESSARY PRECAUTIONS}

In taking precise expansion data, temperature gradients play a large role in the reproducibility of the results. With the interferometer method the specimens can be made small and this greatly reduces gradients. Even so, the temperature indicator and specimens must be in different places and are usually so different in shape, conductivity, and heat capacity that the temperature of the specimens can be appreciably different from that of the indicator. During a uniform heating at only moderate rates, the difference may be unexpectedly large and change considerably with increasing temperature. Holding the furnace for some time at a constant temperature before each reading lowers these differences, but in actual operation does not wholly eliminate them.

For making tests to determine the amount of these gradients, an aluminum cup $5 \mathrm{~cm}$ in height, $4 \frac{1}{2} \mathrm{~cm}$ in diameter, $6 \mathrm{~mm}$ in wall thickness, and with a $2-\mathrm{cm}$ opening in the top, was used. The opening in the top was closed with a fused-quartz window. Within the cup, a porcelain plate $3 \mathrm{~mm}$ in thickness covered the bottom. A fused- 


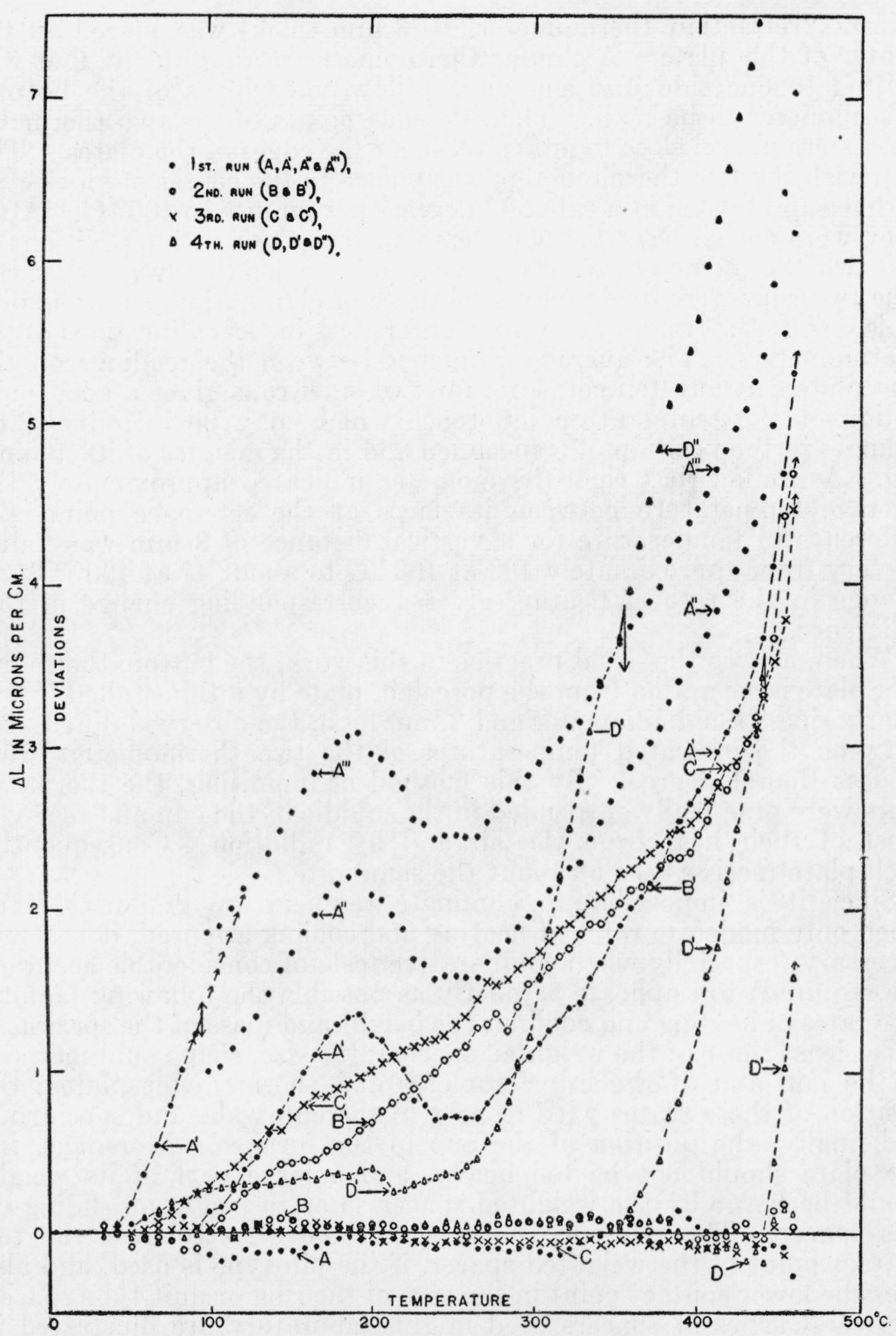

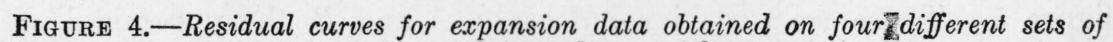
separators from the same glass.

These residuals are the differences between observed results and the probable values for the expansion as determined from the average or preferred data (continuous curve in fig. 3 ).

Curves $A, B, C$, and $D$ are the residuals in the preferred data obtained at reference points over weighted spacers. Curves designated by primed symbols represent deviations of results obtained at median reference points. 
quartz "refraction thermometer" 13 (3 mm thick) was placed on the center of this plate. A similar thermometer resting on the first was shifted to one side just enough to allow the fringes of the bottom thermometer to be read. The reference points of the two thermometers were placed close together and near the edges of the plates. The cup with the two thermometers was placed in the center of an electric furnace and heated at a rate of 3 degrees per minute to $400^{\circ} \mathrm{C}$. After they were cooled back to room temperature, the relative positions of the two thermometers were reversed and the heating was repeated. The two runs were made for the purpose of eliminating, as far as possible, errors that might arise from differences in the calibrations of the thermometers. The average difference between the readings on the two plates, at any temperature, for two such runs gives a good indication of the temperature difference which may be introduced by changes in the position of a specimen and in the manner of its mounting. Assuming that each thermometer indicated approximately the average temperature between its faces at the reference point, the difference in temperature for a vertical distance of $3 \mathrm{~mm}$ was found to vary from approximately $10^{\circ}$ at $100^{\circ} \mathrm{C}$ to about $4^{\circ}$ at $400^{\circ} \mathrm{C}$. A change in the rate of heating gives a corresponding change in this difference.

When, as was the usual practice in this work, the bottom thermometer plate is separated from the porcelain plate by a thin-walled fusedquartz ring $8 \mathrm{~mm}$ in diameter and $3 \mathrm{~mm}$ high, the observed differences between the indicated temperatures of the two thermometers were all less than 1 degree. By this method of mounting, the thermometers were practically suspended in the middle of the cup and received most of their heat from the air and by radiation. Consequently, each plate receive heat at about the same rate.

Since it is impossible to eliminate temperature gradients, even when only moderate rates of heating and cooling are used, it becomes necessary (especially when comparative tests of considerable accuracy are required) to duplicate as nearly as possible the following factors: The rates of heating and cooling; the height and mass of the specimens (or at least those of the weighted spacer); the size, shape, and material of the cup and of the interferometer and thermometer plates; the position of these plates with respect to the cup walls and specimens; and finally, the position of the cup in the furnace. Moreover, the top plate should not be too heavy, and a large part of its weight should be borne by the weighted spacer so as to insure no sliding on this spacer. The reference point should be directly over the top contact point of the weighted spacer, if the pin-type is used, and also over the lower contact point in the case of the ring or unit-type spacer.

Several types of spacers used in this laboratory are illustrated in figure 5. There are two views of each: $A$ and $B$, the pyramidal or pin types; $C$ and $D$, the ring type mentioned in a previous paper, ${ }^{14}$ and the $Y$ - and T-types, represented by $E$ and $F$, respectively. The $T$ - and $Y$-types are recommended for ease in making observations. They are easily constructed and mounted and also are free from tilting. When used between plates having the proper expansivity, these types are also free from surface-film effects after a preheating to $250^{\circ} \mathrm{C}$. 


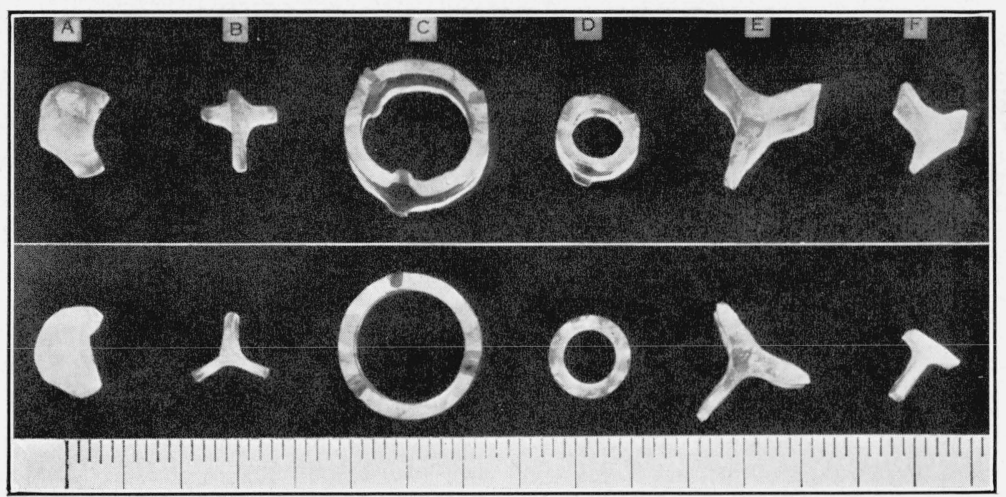

FigURE 5.-Several forms of specimens.

For the types $A$ and $B$ three such tripods are required to support the top interferometer plate, whereas one of the other types is sufficient. The seale is in millimeters. 
By taking all of the precautions mentioned, results on the expansivity of glass can be duplicated by using the interferometer method and any of the above types of spacers to within $1 / 3$ percent, if the expansions exceed 10 fringes and the temperature range of the test is of the order of $100^{\circ} \mathrm{C}$ or more. Of these precautions, properly weighting one spacer and choosing the position of the fiducial point have proved to be most important and have permitted the precision, and also the accuracy, to be increased at least tenfold over that obtainable by most of the previous interferometric procedures. This has been accomplished by the almost complete elimination of the effects caused by the lateral expansions of spacers and plates and by changes in air films. Some of the residual uncertainty is the result of temperature gradients and errors in the temperature determinations. If these sources of error which are also present in all similar procedures, could be eliminated, the precision would be sensibly increased, and the accuracy of the results for the expansivity should closely approach that indicated by the precision.

With all the precautions taken, it is considered possible that the determinations of the temperature range may be in error by as much as 1 degree, even for slow rates of heating. Where rates of 3 or 5 degrees per minute are used, the errors may approach 2 or 3 degrees for a range of $200^{\circ} \mathrm{C}$. In measuring differences between the expansitivity of two or more materials, these errors are to a great extent eliminated, especially if the same apparatus and test conditions are used. Also, they tend to be eliminated in the expansivity results if the thermometer plate is calibrated in the same apparatus and under the same conditions used in the expansion measurements.

Washington, April 29, 1939. 